\title{
The Guarantee Strategy for Reliable Operation of the Precipitation with Mobile Electrode Electrostatic Technology
}

\author{
Shukui Gao', Biao Xie ${ }^{2 *}$ \\ ${ }_{2}^{1}$ Hebei Handan Thermal Power Co., Ltd., Handan, Hebei, China \\ ${ }^{2}$ Anhui Gas Purification Engineering Technology Research Center, Bengbu, Anhui, China \\ Email: bxieb@163.com
}

\begin{abstract}
Research in view of the conventional electrostatic precipitator for ultra fine dust, dust ratio resistance and high viscosity difficult removal, secondary problems such as dust and domestic environmental protection, professional factory introduction, digestion and innovation has been conducted. A new type of electrostatic precipitator, based on the technology of moving electrode, has the following characteristics: low operating cost, high filter processing and small resistance. In order to promote its reliability, the research for mobile motor technology principle and innovative design is made. This paper discusses the moving electrode duster guarantee reliable operation strategy, in order to offer reference to related staff.
\end{abstract}

Keywords: mobile electrode technology, electrostatic precipitation, reliable operation, safeguard strategy

\section{Introduction}

With the continuous development of the urbanization process and the implementation of the sustainable development strategy, China has raised the environmental emission standards, strictly limiting the concentration of dust emission to no more than $10 \mathrm{mg} / \mathrm{m}^{3}$. Conventional electric dust collectors have high costs and heavy workload. There are certain defects in the treatment of cloth bags and high humidity smoke ${ }^{[1]}$. In recent years, new dust removal equipment has been developed, which incorporates mobile electrode technology to make up for the shortcomings of traditional electrostatic precipitators. While reducing operating costs, it reduces daily maintenance workload and can make national emissions without occupying a lot of space meet standard requirements ${ }^{[2]}$. At present, the electrostatic precipitator of moving electrode technology has been tested intermittently, and its dust removal effect has been confirmed. This article discusses the development and design of the mobile electrode electrostatic precipitator, and provides a reference for the reliable operation of the mobile electrode precipitator.

\section{An overview of mobile electrode technology}

\subsection{Working principle of mobile electrode}

The electric field of the mobile electrode is mainly composed of a movable dust collecting plate, a rotating dust cleaning device and a fixed discharge electrode. Generally, when the thickness of the dust collected by the dust collecting plate does not reach the level of back corona, it will move along the moving plate towards the smokeless. The circulating ash hopper is transferred. At this time, the brush will completely remove the dust to ensure the cleaning of the mobile polar plate. In this process, the entire dust removal process is implemented in a dust-free ash hopper, which can avoid the phenomenon of dust flying twice ${ }^{[3]}$. Generally, based on the needs of actual work, different ratios of the moving speed of the polar plate and the angular speed of the rotating brush can be adjusted. These are achieved by frequency conversion stepless speed regulation when faced with different coal dust or different working conditions, which can improve the applicability.

\subsection{Innovation points of mobile electrode technology design}

Different from the traditional electrostatic precipitator, the electrostatic precipitator with mobile electrode technology can realize the efficient collection of high specific resistance dust, which not only does not occupy a lot of space, but also can reduce the application of energy. Usually a mobile electrode plate can play the role of $2 \sim 3$ conventional dust collector electric field, but from the perspective of its power consumption, less than $1 / 2$ of the conventional dust collector. Secondly, under the moving electrode technology, the dust collector can withstand $350^{\circ} \mathrm{C}$ short-term high temperature, has the characteristics of high temperature resistance and corrosion resistance, and has high applicability in various types of dust collection. In the sludge incinerator, coal-fired boiler, metallurgy and other fields are widely used. So the cleaning 
work of the electrostatic precipitator of the mobile electrode technology is mainly carried out in a no-airflow environment, which can not only improve the cleaning effect, but also effectively prevent the dust from flying again ${ }^{[4]}$. In addition, the mobile electrode technology also breaks through the previous aspect ratio design concept when designing the electrostatic precipitator, breaking the site restrictions and providing flexibility.

\subsection{Problems with moving electrodes}

The background of the application of mobile electrode technology is the improvement of environmental protection standards, requiring dust emission concentration less than $30 \mathrm{mg} / \mathrm{m} 3$ in general areas and less than $20 \mathrm{mg} / \mathrm{m} 3$ in key areas. However, the technical form of a spur of the moment and the dust removal effect once aroused the excitement of the academic circle and the industry. However, with the inspection of time, its defects also emerged, mainly including: A has many rotating parts, and the failure rate is high due to fatigue and wear; B has high requirements on product manufacturing quality, installation quality and operation and maintenance level; $\mathrm{C}$ does not solve the secondary dust problem after the cathode is shaken. In view of the problems of some equipment in service, such as lengthening the movement interval time, slowing down the movement speed, lengthening the ash cleaning time, stopping the movement or rotation, or even dismantling and restoring to the conventional electric field, this paper puts forward some specific improvement ideas for discussion, so as to make use of the advantages and circumvent the disadvantages, in order to tap its new vitality.

\section{The mobile electrode combination technology design}

\subsection{Chain design}

The steel carburizing material selected in the chain design link meets the basic requirements of material mechanics and is designed in strict accordance with industry standards. It not only has high strength but also high hardness. Analysis of its safety factor is more than 5 times the actual operating load, and the service life of the chain and the polar plate can reach more than 10 years. The performance process design of the mobile electrode chain is shown in Figure 1. The material used for the chain is $40 \mathrm{Mn}$, which needs to be quenched in the heat treatment process. When it reaches $42 \mathrm{HRC}$ $44 \mathrm{HRC}$, it is adjusted to medium temperature treatment, and tempered at $420^{\circ} \mathrm{C}$. The $40 \mathrm{Cr}$ material is selected for the chain pin, which is first quenched. When it is in 50HRC-55HRC, the temperature is adjusted to $300^{\circ} \mathrm{C}$ for tempering. As the main wear-resistant part of the chain structure, the sleeve is made of 20 \# steel carburized material, after quenching treatment reaches $58 \mathrm{HRC}-62 \mathrm{HRC}$, and then adjust the temperature to $200^{\circ} \mathrm{C}$ for intermediate temperature treatment. The material used for the roller is $40 \mathrm{Cr}$, which is first quenched. When it reaches the 50HRC-55HRC standard, the temperature is adjusted to $300^{\circ} \mathrm{C}$ and tempered ${ }^{[5]}$. As an important part of the surface treatment, the drum shot peening first needs to use the corresponding diameter steel shot to spray the above parts on the surface of the part. At this time, the surface will form a pit of uniform size. The improvement of fatigue strength at the same time can also eliminate internal stress, which is conducive to grinding sharp corners on the chain. In order to improve the riveting strength, the mobile motor technology also increases the diameter of the riveting pin shaft on the basis of the traditional riveting pin shaft. 




Figure 1. Structure diagram of moving electrode chain components

In the operation of welding chain plates and chain plate accessories, carbon dioxide gas is used to protect the welding, and mechanical performance testing and process evaluation are also carried out before the operation. After processing the various parts of the chain, the crystal structure of the chain material has been refined, which can protect the crystal structure, strength and toughness. After the internal stress is eliminated, the service life of the chain can also be extended ${ }^{[6]}$. Generally, the temperature of electric dust removal is controlled at about $150^{\circ} \mathrm{C}$ during operation. Under this temperature condition, the chain will not change significantly. Various parts of the chain are quenched and tempered during the heat treatment. In fact, based on the tempering characteristics of steel Only a few hours before tempering treatment will affect the hardness, and subsequent tempering treatment will not have too much impact on the hardness and internal stress of the steel. Since the crystal structure of each part of the chain tends to be stable, it will not change significantly even if it is placed under the operating temperature of the ESP.

\subsection{Manufacture sprockets in two halves}

It is difficult to replace the dust collector on the traditional chain. This study combined the wear characteristics of the chain roller and the sprocket to comprehensively evaluate the wear of the sprocket and designed a chain roller and sprocket made of $40 \mathrm{Cr}$. In the heat treatment, the hardness of the chain roller needs to be quenched to reach 50HRC-55HRC, and the hardness of the sprocket quenching is preferably 40HRC-45HRC. Compared with the hardness of the sprocket, the hardness of the chain roller is greater, so the general sprocket is first damaged. Under the moving electrode technology, the two half wheels can be rotated through $180^{\circ} \mathrm{C}$ to replace the sprocket ${ }^{[7]}$. At the same time, it is appropriate to increase the width of the meshing teeth of the sprocket, which can enhance the wear resistance of the sprocket and protect the chain, which is conducive to the stable operation of the device.

\subsection{Elimination of mobile electrode swing and thermal displacement}

In practical engineering applications, the running speed of the mobile electrode chain is generally $0 \sim 1.5 \mathrm{~m} / \mathrm{s}$. However, due to the industrial and mining airflow, the chain in the existing mobile electrode will carry a dust collecting plate that will swing during operation, and then the Dust distance and operating parameters have an impact. In response to this problem, a guide wheel was introduced in the chain design, and the guide groove device was installed in the housing. At the same time, the guide wheel and the guide groove are also installed on the lower sprocket shaft, which can prevent the thermal displacement in the running state, so that the mobile electrode dust collector can continue and operate stably ${ }^{[8]}$.

\subsection{Design of cleaning brush}

The cleaning system used in the electrostatic precipitator of moving electrode technology drives the sprocket, chain and gear under the action of the reducer, and connects it with the stainless steel brush to achieve the purpose of cleaning by rotating. During the cleaning process, the displacement of the bolt adjusting bearing can be adjusted, which can make up for the positive pressure of the steel brush on the pole plate. The cleaning brush used is a chain belt mode driven by a reducer, which can drive multiple chains at the same time, and realize the simultaneous operation of multiple cleaning 
brushes for cleaning. The cleaning brush can directly contact with the anode dust collecting plate, so that the anode dust collecting plate can be kept clean ${ }^{[9]}$. The dust collector is suitable for large air volume and temperature according to different situations. Using a reasonable dust removal cycle can not only improve the dust removal efficiency, but also increase the service life.The dust removed from the dust collector can be directly discharged into the trash bin, and the clean air intake fan is discharged.

\section{Application advantages of mobile electrode technology electrostatic precipitator}

Compared with ordinary electrostatic precipitators, the electrostatic precipitator supported by the mobile electrode technology is suitable for a variety of different coal types, which can effectively solve the problem that the traditional precipitator does not meet the standards for the combustion of poor quality and special coal flue gas. Meet the emission requirements of national standards. Secondly, the retrofit of electrostatic precipitator supported by mobile electrode technology requires a small site, and it can be retrofitted even in an environment with a small site and large flue gas emissions, while conventional electric precipitator is difficult to meet the expansion needs. At the same time, the electrostatic precipitator can achieve a better dust removal effect while reducing maintenance costs. It has been proved by practice that the dust collection effect of the mobile electrode is equal to that of the bag-type dust removal, and its dust removal efficiency reaches $99.85 \%$, which can reduce the concentration of the emitted flue gas to $30 \mathrm{mg} / \mathrm{Nm}^{3}{ }^{[10]}$, while in operation and maintenance costs, it is much lower than Baghouse. In addition, the mobile electrode nuclear technology electrostatic precipitator is suitable for flue gas dust removal in many different conditions. Generally, the high temperature, high humidity and high viscosity problems that cannot be solved by the bag type dust collector can be effectively carried out by the electrostatic precipitator. deal with. In the reconstruction project of the electrostatic precipitator with mobile electrode technology, its cost is relatively low. The resistance of the previous bag-type dust collector is relatively large, and the frequency of fan replacement is high. Under the mobile electrode technology, there is no need to replace the fan, which reduces the cost of renovation. From the perspective of the intensity of the transformation work, when the flue gas emissions are the same, the transformation of the mobile electrode only needs to replace the final electric field, and the bag filter needs to replace all the electric field.

\section{Conclusion}

Compared with the conventional electrostatic precipitator, the effective dust collection area of the mobile electrode technology is about $20 \%$ smaller than that of the conventional electrostatic precipitator, but its actual dust collection function is equivalent to 2-3 conventional electric fields. According to the consensus Deutsch efficiency formula, the dust displacement velocity of the mobile electrode technology is larger than that of the conventional electric field, which is the magic power of its vitality.

During the design, factors such as expansion, wear, fatigue, material service life and so on should be fully considered. Sealing must be inspected, all damage must be repaired. It is necessary to improve the reliability of moving and rotating parts so as to make them play out efficiently and stably for a long time and keep them youthful.

Under the guidance of the sustainable development strategy, energy saving, emission reduction as well as the environmental protection standards are becoming more and more stringent. In the past, outdated equipment was unable to meet environmental protection needs. The electrostatic precipitator designed with the support of the mobile electrode technology has distinct advantages in both the use of the environment and the stability of equipment. It can actively promote the technical route of electrostatic precipitator efficiency improvement, replace the conventional bag precipitator, and play its role in energy conservation and emission reduction.

\section{References}

[1] Ren Yan, Kong Chunlin, Liu Jie, et al. Application of pulse enhancement and mobile electrode technology in dust removal transformation of thermal power plants. China Electric Power. 2016; 49(9): 181-184.

[2] Chen Yuanzhao. Retrofit design of electrostatic precipitator based on variable frequency pulse power control.Industrial Instrumentation and Automation. 2019; 14(6): 112-116.

[3] Zhou Chunxiao, Liu Baiqian, Wang Mengqi, et al. DOE experimental analysis of factors affecting the performance of electrostatic precipitator. Thermal Power Generation. 2019; 48 (11): 115-121.

[4] Zhou Chunxiao, Sun Yifan, Jiang Renbao, et al. Suppression effect of blinds on secondary dust emission of electrostatic precipitator. Science Technology and Engineering. 2019; 19(15): 365-371. 
[5] Zhang Jianping, Jiang Zexin, Xu Dacheng. The effect of working voltage on the dust removal efficiency of the same kind of particles in wire-plate electrostatic precipitator under magnetic field environment. Science Technology and Engineering. 2019; 19(25): 392-395.

[6] Yuan Hui, Liu Yanfeng, Song Jinghui, et al. Numerical research on the removal of dust particles by electrostatic precipitator of 1000MW unit. Thermal Power Engineering. 2019; 34(10): 114-121,142.

[7] Wang Xiaolong, Wang Xiaofeng, Zhou Qixin, et al. Comparison of mercury removal performance between bag filter and electrostatic precipitator in cement production line. Cement. 2018; 35(4): 54-59.

[8] Sun Danfeng, Luo Pengzhen, Lu Na, et al. Study on the efficiency of wide-spaced long-barbed electrostatic precipitator to collect simulated dust in highway tunnel. High Voltage Electrical Appliances. 2019; 55(12): 54-60.

[9] Dong Ming, Zhang Yuxuan, Li Sufen, et al. Effect of flue gas velocity on the removal of fine particles in electrostatic precipitator under different polar line structures. Thermal Science and Technology. 2018; 17(1): 41-48.

[10] Li Tingshuai, Wang Qin, Huang Tiefeng, et al. Research on PVA nanofiber based cigarette tar filtration device. Journal of University of Electronic Science and Technology of China. 2019; 48(5): 690-697. 